\title{
Una escala para evaluación del riesgo de caídas en adultos mayores es útil para predecir lesiones
}

\author{
Scale for assessing the risk of falls in older adults is useful for predicting injuries
}

Nilsson M y col. J Am Geriatr Soc 2016;64: 2242-50.

\section{Objetivos}

Investigar el rol de la evaluación del riesgo de lesiones por caídas en adultos mayores mediante la escala de riesgo de caída de Downton (ver tabla 1), para predecir lesiones relacionadas con la caída: traumatismo encefalocraneano (TEC), fractura y muerte.

\section{Diseño, lugar y participantes}

Estudio observacional de cohorte prospectiva realizado en Suecia. Se incluyeron 128.596 adultos mayores (edad media 82,4 $+/-$ 7,8 años) a quienes se les realizó la evaluación del riesgo de caídas usando la escala de Downton. La información sobre TEC, fracturas de cadera y mortalidad por causa de una caída fue recolectada del Registro Sueco de pacientes y del Registro de Causa de Muerte. El rol predictivo del riesgo de lesión usando la escala de Downton se calculó utilizando los modelos de regresión de Poisson con edad, sexo, altura, peso y comorbilidades como covariables, teniendo en cuenta el momento de la ocurrencia de los eventos o el final del estudio.

\section{Resultados}

Durante un seguimiento medio de 253 días, 15.299 participantes tuvieron una lesión relacionada con una caída, 2.864 presentaron TEC, 2.557 sufrieron una fractura de cadera, y 23.307 murieron. El subgrupo de alto riesgo de caída categorizado mediante la escala de Downton (con un valor mayor o igual a 3) predeciría de forma independiente y ajustado a variables de comorbilidad un mayor riesgo de sufrir lesiones por caída, fractura de cadera o

Tabla 2: Valor predictivo de riesgo de caídas, fractura de cadera, traumatismo de cráneo y mortalidad, según el puntaje de la escala de riesgo Dowtown*
TEC, así como de morir por cualquier tipo de causa (ver tabla 2). Según un análisis por subgrupos de edad, esta escala de riesgo predeciría en mayor medida los eventos medidos en personas de 70 años comparándolos con los de 90 años (para TEC: HR 1,29 [IC 95\% 1,21 a 1,36] vs. 1,08 [1,04 a 1,11], respectivamente; para fractura de cadera: 1,41 [1,30 a 1,53] vs 1,08 [1,05 a 1,11]).

Tabla 1. Escala de riesgo de caídas de Dowtown*

\begin{tabular}{l|l|c}
\hline \multicolumn{1}{c|}{ Variable de medición } & \multicolumn{1}{|c}{ Respuesta } & Valor \\
\hline Caídas previas & No & 0 \\
& $\mathrm{Si}$ & 1 \\
\hline Medicamentos & Ninguno & 0 \\
& Tranquilizantes-sedantes & 1 \\
& Hipotensores (no diuréticos) & 1 \\
& Anti parkinsonianos & 1 \\
& Antidepresivos & 1 \\
& Otros medicamentos & 1 \\
\hline Déficits sensoriales & Ninguno & 0 \\
& Alteraciones visuales & 1 \\
& Alteraciones auditivas & 1 \\
\hline Estado mental & Extremidades (ictus) & 0 \\
& Orientado & 1 \\
\hline Deambulación & Confuso & 0 \\
& Normal & 1 \\
& Segura con ayuda & 1 \\
\hline & Insegura con ayuda/sin ayuda & \\
& Imposible & \\
\hline
\end{tabular}

*Interpretación del puntaje: valor igual o mayor a 3: alto riesgo; valor de 1 a 2: mediano riesgo; valor de 0 a 1: bajo riesgo.

\begin{tabular}{|c|c|c|}
\hline Variable medida & $\begin{array}{c}\text { Puntaje mayor o igual a } 3^{*} \\
\text { HR (IC 95\%) }\end{array}$ & $\begin{array}{c}\text { Puntaje mayor o igual a } 7^{*} \\
\text { HR (IC 95\%) }\end{array}$ \\
\hline Lesión por caída & $1,43(1,39$ a 1,49$)$ & $1,36(1,26$ a 1,46$)$ \\
\hline Fractura de cadera & $1,51(1,38$ a 1,66$)$ & $1,51(1,29$ a 1,79$)$ \\
\hline Traumatismo de cráneo & $1,12(1,03$ a 1,22$)$ & $1,32(1,13$ a 1,55$)$ \\
\hline Mortalidad por todas las causas & $1,39(1,35$ a 1,43$)$ & $1,44(1,37$ a 1,53$)$ \\
\hline
\end{tabular}

HR: hazard ratio; IC 95\%: intervalo de confianza del 95\%. *Ajuste utilizando modelo de Cox según las siguientes variables: edad, sexo, peso, talla, anemia, diabetes mellitus tipo 1 y 2, enfermedad de alzheimer, cataratas, hipertensión arterial, insuficiencia cardiaca, enfermedad coronaria, fibrilación auricular, accidente cerebrovascular, enfermedad pulmonar obstructiva crónica, asma, artrosis de rodilla o cadera, insuficiencia renal crónica. \#Tomando como referencia valores de la escala de 1 o 2 puntos.

\section{Conclusión}

La evaluación del riesgo de caídas en adultos mayores, mediante la escala de Downton puede predecir lesiones relacionadas, incluyendo fractura de cadera, traumatismo de cráneo, y mortalidad por todas las causas tanto en hombres como en mujeres. Esto permitiría identificar quienes se beneficiarían de intervenciones para reducción el riesgo de caídas. 


\section{Comentario}

Las caídas en los adultos mayores son frecuentes. Aproximadamente 1 de cada 3 adultos mayores sufre una caída en el transcurso de un año ${ }^{1}$. Factores de riesgo tales como la edad, el antecedente de haber sufrido caídas previas, la medicación inapropiada y la polifarmacia, los trastornos en la marcha y las alteraciones cognitivas y del ánimo aumentan este riesgo ${ }^{2}$. La importancia de la caída, no radica en el evento en sí mismo, sino en sus consecuencias. Según estadísticas foráneas aproximadamente $15 \%$ de los adultos mayores que se caen sufren alguna lesión, 10\% requiere atención médica y aproximadamente 5\% sufre una fractura ${ }^{2}$. Otras consecuencias menos severas son las relacionadas con el miedo a caer nuevamente. En la práctica habitual no está recomendado en forma homogénea el tamizaje de caídas en adultos mayores, pero algunas sociedades científicas como la Fuerza de Tareas Preventiva de los EE.UU. (USPSTF, por sus iniciales en inglés $)^{3}$ y la Asociación Norteamericana de Geriatría o (AGS, por sus iniciales en inglés) ${ }^{4}$ recomiendan indagar al menos una vez al año a los mayores de 65 años si sufieron caídas en el año previo. Ante la respuesta positiva de- bería realizarse una valoración funcional, a través de la prueba "levántate y anda" o la batería breve de evaluación funcional de Guralnik (también conocida como SPPB). Cuando estas pruebas son patológicas debería realizarse una valoración geriátrica integral (VGI) enfocada en factores de riesgos de caer, escalas de funcionalidad, cognición y ánimo, examen físico para detectar patologías médicas que puedan ocasionarlos, y a partir de allí diseñar un plan de trabajo enfocado en la prevención. Ante un paciente con riesgo de caída se deberá apuntar a modificar aquellos factores de riesgo modificables y orientar el tratamiento a los hallazgos objetivados en la VGI. Al momento actual, las medidas que han demostrado ser eficaces para prevenir las caídas incluyen el ejercicio físico (Grado B) y la modificación del entorno (Grado $\mathrm{C})^{2}$. La USPSTF3 desaconseja la administración de vitamina $\mathrm{D}$ en dosis diaria de $1000 \mathrm{UI}$ de colecalciferol como medida de prevención (Grado D), aunque algunas sociedades científicas la mantienen. Otras posibles intervenciones centradas en la persona no cuentan con recomendaciones a nivel poblacional.

Fabiana Giber [ Medicina Geriátrica. Servicio de Clínica Médica del Hospital Italiano de Buenos Aires. fabiana.giber@ hospitalitaliano.org.ar]

Giber F. Una escala para evaluación del riesgo de caídas en adultos mayores es útil para predecir lesiones. Evid Actual Pract Ambul 2018;21(3):8384. Comentado de: Nilsson M y col. Fall Risk Assessment Predicts Fall-Related Injury, Hip Fracture, and Head Injury in Older Adults. J Am Geriatr Soc 2016;64: 2242-50. PMID: 2768975.

\section{Referencias}

1. Karlsson MK. y col. Prevention of falls in the elderly: a review. Scand J Public Health. 2013 Jul;41(5):442-54. PMID: 23554390.

2. Pfortmueller CA y col. Reducing fall risk in the elderly: risk factors and fall prevention, a systematic review. Minerva Med. 2014 Aug;105(4):275-81. PMID: 24867188

3. US Preventive Services Task Force, Grossman DC, y col. Interventions to Prevent Falls in Community-Dwelling Older Adults: US Preventive Services Task Force Recommendation Statement. JAMA. 2018 Apr 24;319(16):1696-1704.

4. Giber F. Caídas en Adultos mayores. En: El chequeo después de los 65. Buenos Aires: del Hospital ediciones; 212-227; 2013.

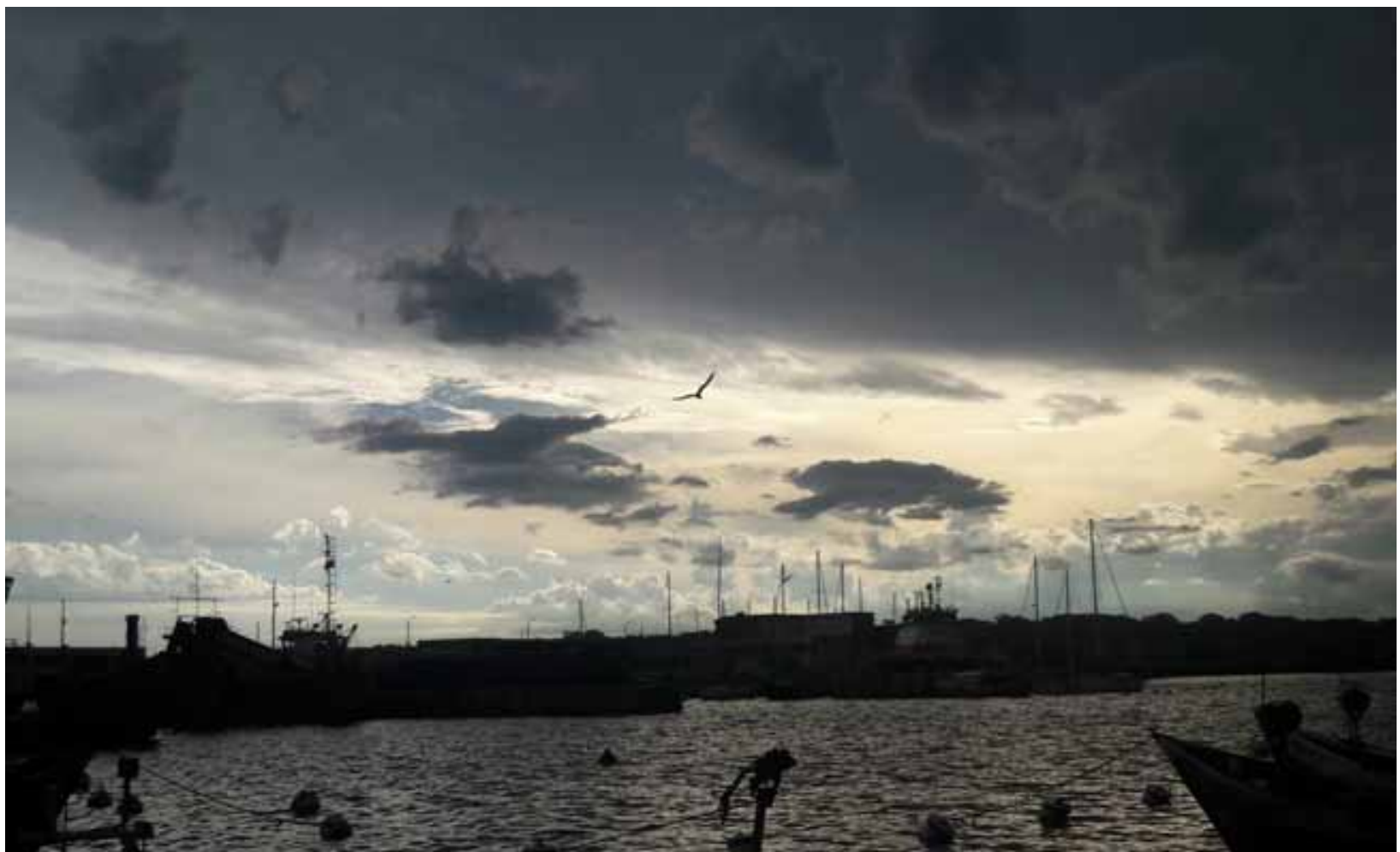

Cortesía de Silvia Spina 